\title{
DEVELOPING SUPPLY CHAIN NETWORK WITH PIECEWISE LINEAR TRANSPORTATION COST FOR A SMALL-AND-MEDIUM ENTERPRISE IN CILEGON
}

\author{
Bobby Kurniawan ${ }^{1}$, Ade Irman', Akbar Gunawan1, Ani Umyati ${ }^{1}$, Evi Febianti ${ }^{1}$, Nuraida Wahyuni ${ }^{1}$, \\ Putro Ferro Ferdinant $^{1}$, Ratna Ekawati ${ }^{1}$, Fellek Getu Tadesse ${ }^{2}$ \\ ${ }^{1}$ Department of Industrial Engineering, Sultan Ageng Tirtayasa University, Indonesia \\ ${ }^{2}$ School of Mechanical and Industrial Engineering, Ethiopian Institute of Technology-Mekelle, Ethiopia \\ e-mail:b.kurniawan@untirta.ac.id
}

\begin{abstract}
This study proposed a supply chain network for determining suppliers' location in which the transportation costs are a piecewise linear function. The supply chain network consists of a production facility, suppliers, and customers. These types of costs are found in the fields of transportation, logistics, and purchasing discount. First, the supply chain network is formulated as the mixed-integer nonlinear programming (MINLP) because piecewise linear transportation cost makes the model non-linear. Then, the model is transformed into a mixed-integer programming (MIP) model using the convex-combination method to overcome this nonlinearity. The model was used for solving the problem faced by a small and medium enterprise (SME) in Cilegon. The MIP was solved using the CPLEX software. Sensitivity analysis was carried to provide the SME with several alternatives in handling the suppliers' location problem.
\end{abstract}

Keywords: Supply chain network; piecewise linear function; convex-combination method; small and medium enterprise

Received: 2021-05-07; Revised: 2021-07-22; Accepted: 2021-07-23

\section{INTRODUCTION}

This study aims to determine the problem of determining the location of the supplier of a Small-and-Medium Enterprise (SME) in the Cilegon area. With growing demand, the business owner feels the need to increase production capacity and open several distribution centers. Therefore, it is necessary to determine a location that can cover a large market and minimal shipping costs. A third party carries out the delivery of goods through a contract system. In this contract, a discount is applied for the number of goods sent. The discount makes SME chooses these third parties as business partners.

The problem is modeled into determining the distributor's location by considering the transportation costs of a piecewise linear function. Transportation costs in the form of a piecewise linear function are widely applied in the real world [1], [2]. For example, this function can be found in discounts on goods [3], [4]. The more the number of goods purchased by the consumer, the more significant the price reduction received.
This study is different from other studies, which assume that transportation costs are constant [5-12]. Conversely, the transportation costs in this study are not constant but a piecewise linear function. A piecewise linear function is a composite function consisting of more than one straight line (line segment). This function is linear for a particular domain, but overall, this function is not linear. Thus, the location determination model becomes non-linear [13]. As a result, this study's problem is more complicated than in previous studies [14].

Several studies have proposed several ways of dealing with nonlinearity. Lagrange decomposition is used to determine where a single location can cover multiple request locations [15]. A greedy adaptive algorithm was developed to determine the location of stochastic demand [16]. Holmberg proposed an approach based on Bender's decomposition to solve the location problem [17]. Other research used a dynamic programming approach to tackle the nonlinearity of the piecewise linear function [18]. A clustering technique was used to solve the 
warehouse location problem [19]. The branchand-cut algorithm is proposed to solve the nonconvex and non-linear model [20], [21].

In this study, the convex-combination method (CCM) is used to change the non-linear form to the linear form [22]. The CCM transforms the nonlinear form into a linear form by reformulating the piecewise linear function into an integer constraint. After linearization was carried out by converting the problem into an integerprogramming model, the CPLEX software was used to find the optimal solution. Furthermore, sensitivity analysis is used to determine total cost changes due to changes in several model parameters.

\section{METHODS}

Quantitative research methods are used in this research. First of all, the problem is formulated. In the next section, the general model for determining the location (facility location problem) is discussed. Furthermore, model development is used to solve problems. The piecewise linear function is discussed next. Finally, this section also discusses the design of numerical experiments.

\section{Problem Formulation}

The problem discussed in this study is the extension of the capacitated facility location problem. The formulation of the problem is as follows.

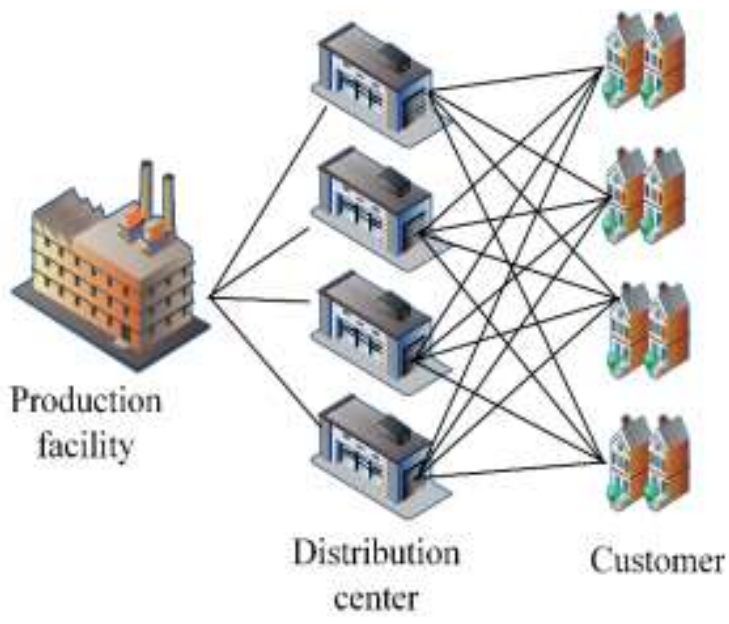

Figure 1. The proposed supply chain network of the SME
An SME in Cilegon has one production facility. With the increasing demand, the SME plans to open several distribution centers (DC) to distribute the goods. To open a DC requires a fixed investment fee. The goods stored in DC will fulfill requests from customers. Third-party transportation companies carry out transfers from a production facility to $\mathrm{DC}$ and shipments from DC to customers.

Based on the contract with the transportation company, shipping costs will be discounted according to the number of products sent. Discounts are categorized into several modes or types of discounts directly influenced by the number of goods transported. For example, suppose a transportation company offers three discount modes. The first mode is the transportation cost for one unit of product is IDR $100.00 / \mathrm{km}$ for the number of shipments between $0-10$ units. The second mode is the transportation fee of IDR $75.00 / \mathrm{km}$ for $10-20$ units shipments. The last mode is the transportation fee, which is IDR $50.00 / \mathrm{km}$ for more than 20 units. The SME conducts research to determine how many suppliers will be opened and the number of goods sent through these suppliers. Figure 1 illustrates the supply chain network that the SME will make.

\section{Mathematical model}

The notations used to model the problem faced by the SME are as follows.

$$
\begin{array}{cl}
\frac{\text { Index }}{i} & : \text { Index of the production facility } \\
j & : \text { Index of distribution center } \\
k & : \text { Index of customer } \\
l & : \text { Index of discount mode } \\
\text { Parameters } \\
\hline N & : \text { The number of production facilities } \\
M & : \text { The number of distribution centers } \\
K & : \text { The number of customers } \\
L & : \text { The number of discount modes } \\
a_{i j l} & : \text { The minimum quantity of products } \\
& \text { sent from production facility } i \text { to } \\
& \text { distribution center } j \text { using discount } \\
& \text { model } \\
a_{i j(l+1)} & : \text { The maximum quantity of products } \\
& \text { sent from production facility } i \text { to } \\
& \text { distribution center } j \text { using discount } \\
& \text { model } \\
\lambda_{i j l} & : \text { The weight of discount model if } \\
& \text { used to deliver products from } \\
& \text { production facility } i \text { to distribution } \\
& \text { center } j
\end{array}
$$




\begin{tabular}{|c|c|}
\hline & \\
\hline$c_{j k l}$ & $\begin{array}{l}\text { : The minimum quantity of products } \\
\text { sent from distribution center } j \text { to } \\
\text { customer } k \text { using discount model }\end{array}$ \\
\hline$c_{j k(l+1)}$ & $\begin{array}{l}\text { : The maximum quantity of products } \\
\text { sent from distribution center } j \text { to } \\
\text { customer } k \text { using discount model }\end{array}$ \\
\hline$\mu_{j k l}$ & $\begin{array}{l}\text { : The weight of discount model if } \\
\text { used to deliver products from } \\
\text { distribution center } j \text { to customer } k\end{array}$ \\
\hline$u_{i}$ & $\begin{array}{l}\text { : Production capacity of production } \\
\text { facility } i\end{array}$ \\
\hline$v_{j}$ & $\begin{array}{l}\text { : Storage capacity of distribution } \\
\text { center } j\end{array}$ \\
\hline$d_{k}$ & : Demand of customer $k$ \\
\hline$g_{j}$ & $\begin{array}{l}\text { : Investment fee to open distribution } \\
\text { center } j\end{array}$ \\
\hline$f$ & : Transportation cost function \\
\hline ecis & variables \\
\hline$x_{j}$ & $\begin{array}{l}: 1 \text { if distribution center } j \text { is open, } 0 \\
\text { otherwise }\end{array}$ \\
\hline$y_{i j}$ & $\begin{array}{l}: \text { Product flow from production facility } \\
i \text { to distribution center } j\end{array}$ \\
\hline$z_{j k}$ & $\begin{array}{l}\text { Product flow from distribution center } \\
j \text { to customer } k\end{array}$ \\
\hline$b_{i j l}$ & $\begin{array}{l}: 1 \text { if products are sent from } \\
\text { production facility } i \text { to distribution } \\
\text { center } j \text { using discount model, } 0 \\
\text { otherwise }\end{array}$ \\
\hline$e_{j k l}$ & $\begin{array}{l}: 1 \text { if products are sent from } \\
\text { distribution center } j \text { to customer } k \\
\text { using discount model, } 0 \text { otherwise }\end{array}$ \\
\hline
\end{tabular}

The supply-chain network for determining the distributors' location with piecewise linear transportation costs is modeled as mixed integer non-linear programming (MINLP) as follows.

$$
\begin{aligned}
\min & \sum_{i=1}^{i=N} \sum_{j=1}^{j=M} s_{i j} f\left(y_{i j}\right) \\
& +\sum_{j=1}^{j=M} g_{j} x_{j} \\
& +\sum_{j=1}^{j=M} \sum_{k=1}^{k=K} t_{j k} f\left(z_{j k}\right)
\end{aligned}
$$

s.t.

$$
\begin{aligned}
& \sum_{j=1}^{j=M} y_{i j} \leq u_{i}, 1 \leq i \leq N \\
& y_{i j} \leq v_{j} x_{j}, 1 \leq i \leq N, 1 \leq j \leq M \\
& \sum_{j=1}^{j=M} z_{j k}=d_{k}, 1 \leq k \leq K \\
& \sum_{k=1}^{k=K} z_{j k} \leq v_{j} x_{j}, 1 \leq j \leq M \\
& \sum_{i=1}^{i=N} \sum_{j=1}^{j=M} y_{i j}=\sum_{j=1}^{j=M} \sum_{k=1}^{k=K} z_{j k}
\end{aligned}
$$

Eq. (1) is the objective function that consists of three parts. The first term represents the transportation expenses for delivering goods from distribution centers to consumers. The second term is the fixed cost of opening a distribution center. Meanwhile, the third term is transportation cost for sending products from the production facility to distribution centers. In the first and second terms, the transportation cost $\boldsymbol{f}$ is a piecewise linear function. Eq. (2) states that the flow of products sent from the production facility to distribution centers does not exceed its capacity. Eq. (3) ensures that the production facility's product flow to the distribution centers does not exceed its storage capacity. Eq. (4) states that all distribution centers' flow is the same as consumer demand. Eq. (5) guarantees no product flow from a distribution center to the consumer if it is not opened. Eq. (6) guarantees that the total product flow from the production facility to the distribution centers equals the total product flow from the distribution centers to the consumer.

\section{Piecewise Transportation Cost}

Transportation cost depends on the number of products shipped. The transportation cost function is expressed as a piecewise linear function, where the function depends on the product transported. Let $y_{i j}$ be the number of products transported from production facility $i$ to distribution center $j$ and $f\left(y_{i j}\right)$ be the transportation cost. Figure 2 illustrates the transportation costs from production facility $i$ to distribution center $j$ with three discount modes $(L=3)$.

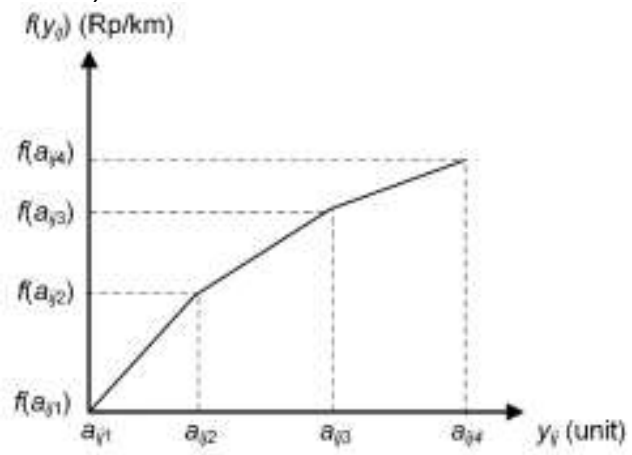

Figure 2. Piecewise transportation cost from production facility $i$ to distribution $j$

From Figure 2, the transportation cost function is linear for each discount mode but not linear as a whole. Each discount mode is a straight line bounded by the minimum value and maximum value. The non-linear transportation cost function can be transformed into a linear form using the convex-combination method. 
Suppose $y_{i j}$ is in the first discount mode $\left(a_{i j 1} \leq y_{i j} \leq a_{i j 2}\right)$, then $y_{i j}$ can be expressed as $y_{i j}=\lambda_{i j 1} a_{i j 1}+\left(1-\lambda_{i j 1}\right) a_{i j 2}$, with $0 \leq \lambda_{i j 1} \leq 1$. However, $y_{i j}$ can also be in the second and third discount modes, Therefore, $y_{i j}$ can be generalized to $y_{i j}=\lambda_{i j 1} a_{i j 1}+\left(1-\lambda_{i j 1}\right) a_{i j 2}+$ $\lambda_{i j 2} a_{i j 2}+\left(1-\lambda_{i j 2}\right) a_{i j 3}+\lambda_{i j 3} a_{i j 3}+(1-$

$\left.\lambda_{i j 3}\right) a_{i j 4}, \lambda_{i j l} \leq 1$, and at most two adjacent $\lambda_{i j l}$ can be positive. Thus, $y_{i j}$ in general can be stated as follows.

$$
\begin{gathered}
\sum_{l=L 1}^{l=L+1} \lambda_{i j l} a_{i j l}=y_{i j}, 1 \leq i \leq N, 1 \leq j \leq \\
M \sum_{l=1}^{l=L+1} \lambda_{i j l}=1,1 \leq i \leq N, 1 \leq j \leq M \\
\lambda_{i j l} \leq b_{i j(l-1)}+b_{i j l}, 1 \leq i \leq N, 1 \leq j \leq \\
M, 1 \leq l \leq L+1 \\
\sum_{l=1}^{l=L} b_{i j l}=1,1 \leq i \leq N, 1 \leq j \leq M
\end{gathered}
$$

In the same way, $f\left(y_{i j}\right)$ can be expressed as the convex combination of discount modes as follows.

$$
\sum_{l=1}^{l=L+1} \lambda_{i j l} f\left(a_{i j l}\right)=f\left(y_{i j}\right), 1 \leq i \leq
$$

\section{$N, 1 \leq j \leq M$}

For the number of products and transportation costs from supplier $j$ to customer $k$, the number of products shipped $z_{j k}$ and the transportation costs $f\left(z_{j k}\right)$ is carried out in the same way. Thus, the variables $z_{j k}$ and $f\left(z_{j k}\right)$ are stated as follows.

$$
\begin{gathered}
\sum_{l=1}^{l=L+1} \mu_{j k l} c_{j k l}=z_{j k}, 1 \leq j \leq M, 1 \leq \\
k \leq K \\
\sum_{l=1}^{l=L+1} \mu_{j k l}=1,1 \leq j \leq M, 1 \leq k \leq K \\
\mu_{j k l} \leq e_{j k(l-1)}+e_{j k l}, 1 \leq j \leq M, 1 \leq \\
k \leq K, 1 \leq l \leq L+1 \\
\sum_{l=1}^{l=L} e_{j k l}=1,1 \leq j \leq M, 1 \leq k \leq K \\
\quad \sum_{l=1}^{l=L+1} \mu_{j k l} f\left(c_{j k l}\right)=f\left(z_{j k}\right), 1 \leq j \leq \\
M, 1 \leq k \leq K
\end{gathered}
$$

In the next step, we substitute $y_{i j}$ in Eqs. (1)(6) with Eq. (7), $z_{j k}$ with Eq. (12), $f\left(y_{i j}\right)$ with Eq. (11), $f\left(z_{j k}\right)$ with Eq. (16) and augmented Eqs. (8)-(10) and (12)-(15) to the model. As a result, the supply chain model becomes as follows.

$$
\begin{aligned}
& \min \sum_{i=1}^{i=N} \sum_{j=1}^{j=M} \sum_{l=1}^{l=L+1} s_{i j} \lambda_{i j l} f\left(a_{i j l}\right) \\
& +\sum_{j=1}^{j=M} g_{j} x_{j} \\
& +\sum_{j=1}^{j=M} \sum_{k=1}^{k=K} \sum_{l=1}^{l=L+1} t_{j k} \mu_{j k l} f\left(c_{j k l}\right)
\end{aligned}
$$

3s.t.

$$
\begin{gathered}
\sum_{j=1}^{j=M} \sum_{l=1}^{l=L+1} \lambda_{i j l} a_{i j l} \leq u_{i}, 1 \leq i \leq N \\
\sum_{l=1}^{l=L+1} \lambda_{i j l} a_{i j l} \leq v_{j} x_{j}, 1 \leq i \leq N, 1 \leq \\
j \leq M \\
\sum_{j=1}^{j=M} \sum_{l=1}^{l=L+1} \mu_{j k l} c_{j k l}=d_{k}, 1 \leq k \leq \\
K \sum_{k=1}^{k=K} \sum_{l=1}^{l=L+1} \mu_{j k l} c_{i j l} \leq v_{j} x_{j}, 1 \leq j \leq \\
M \quad \sum_{i=1}^{i=N} \sum_{j=1}^{j=M} \sum_{l=1}^{l=L+1} \lambda_{i j l} a_{i j l}= \\
\sum_{j=1}^{j=M} \sum_{k=1}^{k=K} \sum_{l=1}^{l=L+1} \mu_{j k l} c_{j k l}
\end{gathered}
$$

Thus, the new model consists of Eqs. (17)(22), (8)-(10), (13)-(15). The new model can be solved by CPLEX.

\section{Data Collection}

The data were collected by observation from the SME. The data collected include 1) production facility data, 2) distribution center data, 3) customer data. The data regarding the supply chain network are as follows. The number of production facilities is $N=1$, the number of distribution centers $M=4$, and the number of customers $K=6$. The transportation company provides three discount modes $L=3$ with the transportation cost function (in thousands of IDR per $\mathrm{km}$ ) in Eq. (23).

$$
f(q)=\left\{\begin{array}{c}
25+0.25 q, 0 \leq q \leq 100 \\
500+0.15 q, 100 \leq q \leq 200 \\
850+0.1,200 \leq q \leq 300
\end{array}\right.
$$

The production capacity per month of the production facility is 1000 units per month. Meanwhile, the demand from all customers is around 500 units. The data regarding suppliers are presented in Table 1. The data consists of distance from the production facility, investment cost to open distribution center, and the storage capacity.

Table 1. Distribution center data

\begin{tabular}{cccc}
\hline Location & $\begin{array}{c}\text { Distance } \\
\text { from } \\
\text { production } \\
\text { facility } \\
\text { (km) }\end{array}$ & $\begin{array}{c}\text { Investment } \\
\text { cost } \\
\text { (000 IDR) }\end{array}$ & $\begin{array}{c}\text { Storage } \\
\text { capacity } \\
\text { (unit) }\end{array}$ \\
\hline P1 & 14 & 200,000 & 200 \\
P2 & 30 & 150,000 & 200 \\
P3 & 38 & 100,000 & 150 \\
P4 & 36 & 75,000 & 100 \\
\hline
\end{tabular}




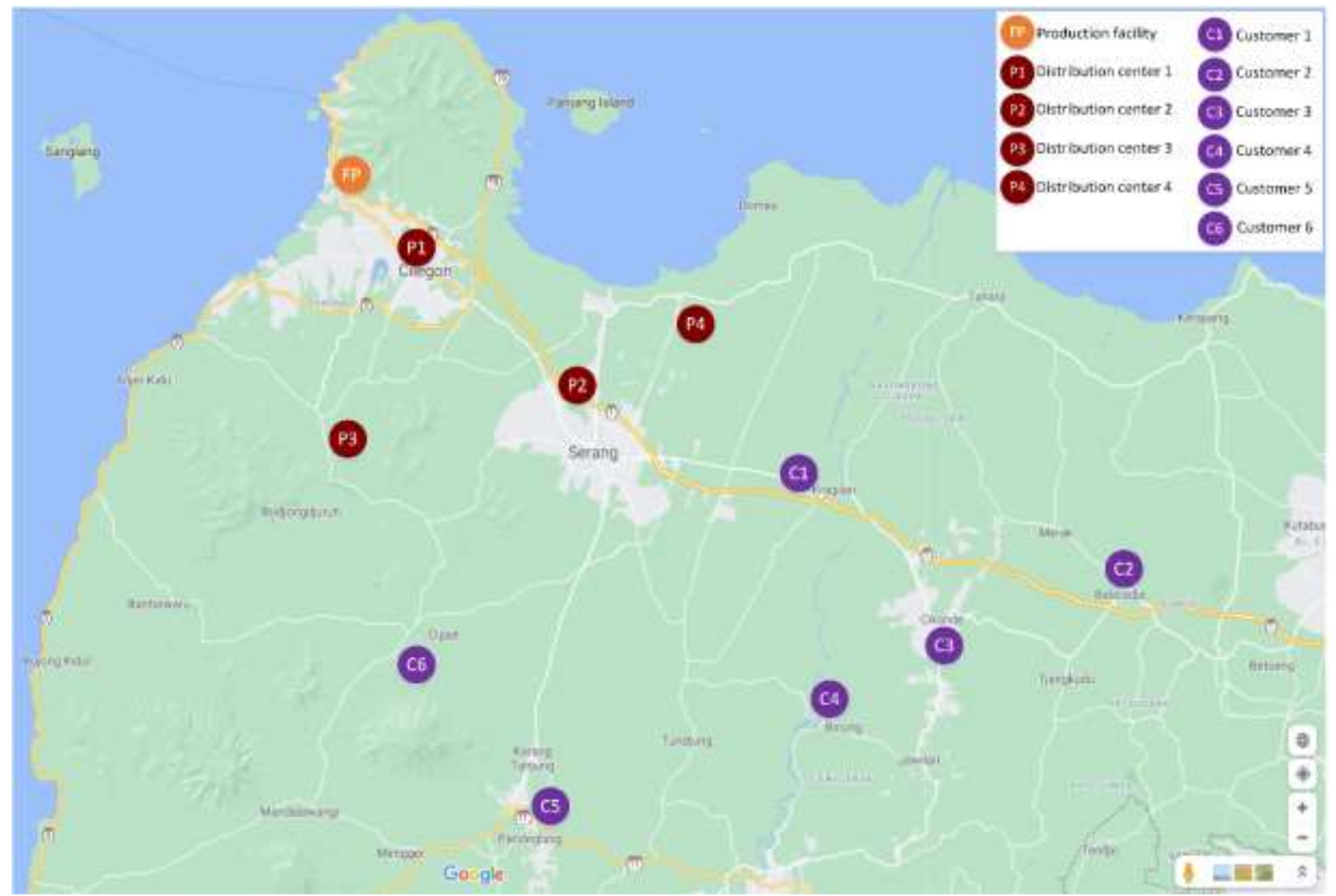

Figure 3. The map of the supply chain network

Figure 3 depicts the locations of the production facility (FP), distribution centers (P1$\mathrm{P} 4)$, and customers (C1-C6). The location of FP is located around the Merak area. The location of distribution centers are Cilegon (P1), Taktakan (P2), Mancak (P3), and Pontang (P4). Currently, there are four locations as the candidate for distribution centers.

Table 2 shows the customer data that consists of its location and demand. The demand is obtained from SME's forecasting.

The locations of customers are Kragilan (C1), Balaraja (C2), Cikande (C3), Binung (C4), Pandeglang (C5), and Cipait (C6). The distance from the distribution center and customer are shown in Table 3.

Table 2. Customer data

\begin{tabular}{clc}
\hline No & \multicolumn{1}{c}{ Location } & Demand (unit) \\
\hline 1 & C1 (Kragilan) & 62 \\
2 & C2 (Balaraja) & 115 \\
3 & C3 (Ciaknde) & 181 \\
4 & C4 (Binung) & 29 \\
5 & C5 (Pandeglang) & 73 \\
6 & C6 (Cipait) & 23 \\
\hline
\end{tabular}

Table 3. Distance matrix from distribution center to customers $(\mathrm{km})$

\begin{tabular}{ccccccc}
\hline Dist. & \multicolumn{7}{c}{ Customers } \\
center & C1 & C2 & C3 & C4 & C5 & C6 \\
\hline P1 & 30 & 46 & 40 & 41 & 38 & 33 \\
P2 & 21 & 41 & 33 & 37 & 33 & 23 \\
P3 & 42 & 66 & 54 & 57 & 46 & 18 \\
P4 & 26 & 46 & 37 & 38 & 36 & 31 \\
\hline
\end{tabular}

\section{Solution Method}

Once the mathematical model has been developed and data has been collected, we seek the solution to the problem using CPLEX software. First, the model (Eqs. (17)-(22), (8)(10), (13)-(15)) are coded using OPL modelling language and the data collected are inputted as parameters. Then, we solve the code using CPLEX software. The code and CPLEX software are running on a hexa-core computer using $8 \mathrm{~GB}$ of RAM.

\section{RESULTS AND DISCUSSION}

Table 4 presents the results from the CPLEX software. The results showed that P1, P2, and P4 
are opened as distribution centers, whereas location P3 is not opened. The production facility sends 183 units of product to P1, 200 units to P2, and 100 units to P4. Distribution center P1 sends 100 units to $\mathrm{C} 2$, ten units to $\mathrm{C} 4$, and 73 units to C5. P2 sends 62 units of product to $\mathrm{C} 1,15$ units of it to C2, 100 units of it to C3, and 23 units to C6. Finally, P4 sent 81 units of products to $\mathrm{C} 3$ and 19 units of products to $\mathrm{C} 4$. The total cost required is IDR $458,254,250$. The computation time required is 0.05 seconds.
Table 4. Optimal product flow (unit)

\begin{tabular}{ccccccc}
\hline Dist. & \multicolumn{7}{c}{ Customers } \\
center & C1 & C2 & C3 & C4 & C5 & C6 \\
\hline P1 & 0 & 100 & 0 & 10 & 73 & 0 \\
P2 & 62 & 15 & 100 & 0 & 0 & 23 \\
P3 & 0 & 0 & 0 & 0 & 0 & 0 \\
P4 & 0 & 0 & 81 & 19 & 0 & 0 \\
\hline
\end{tabular}

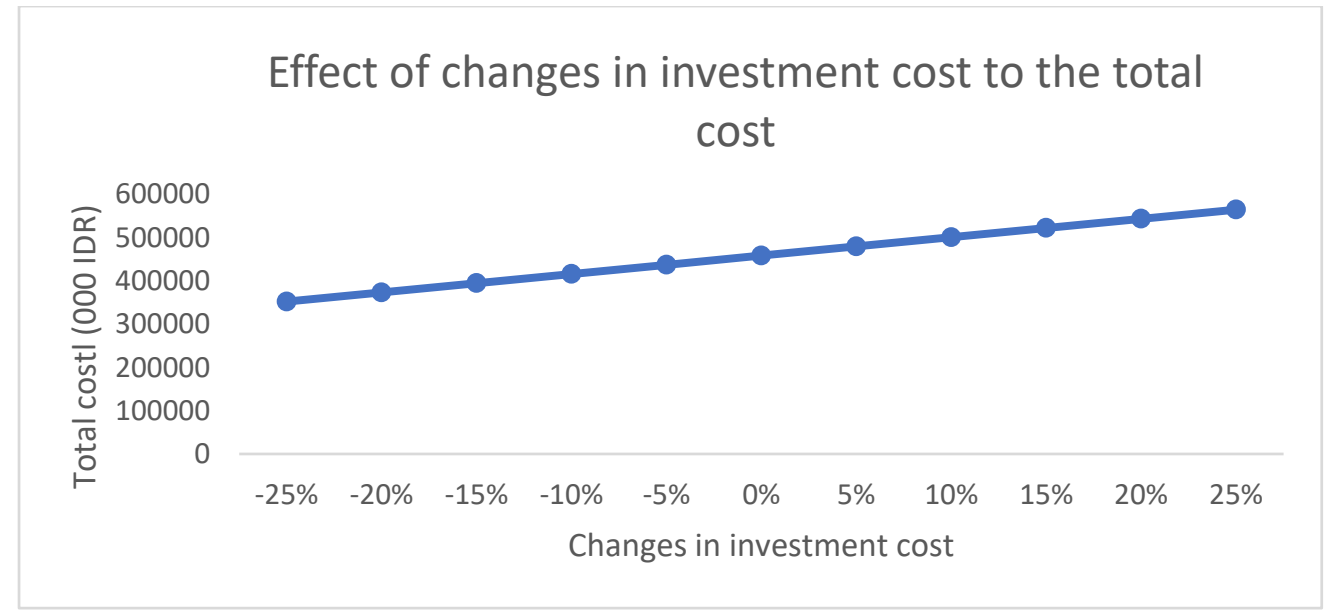

Figure 4. Effect of investment cost to the total cost

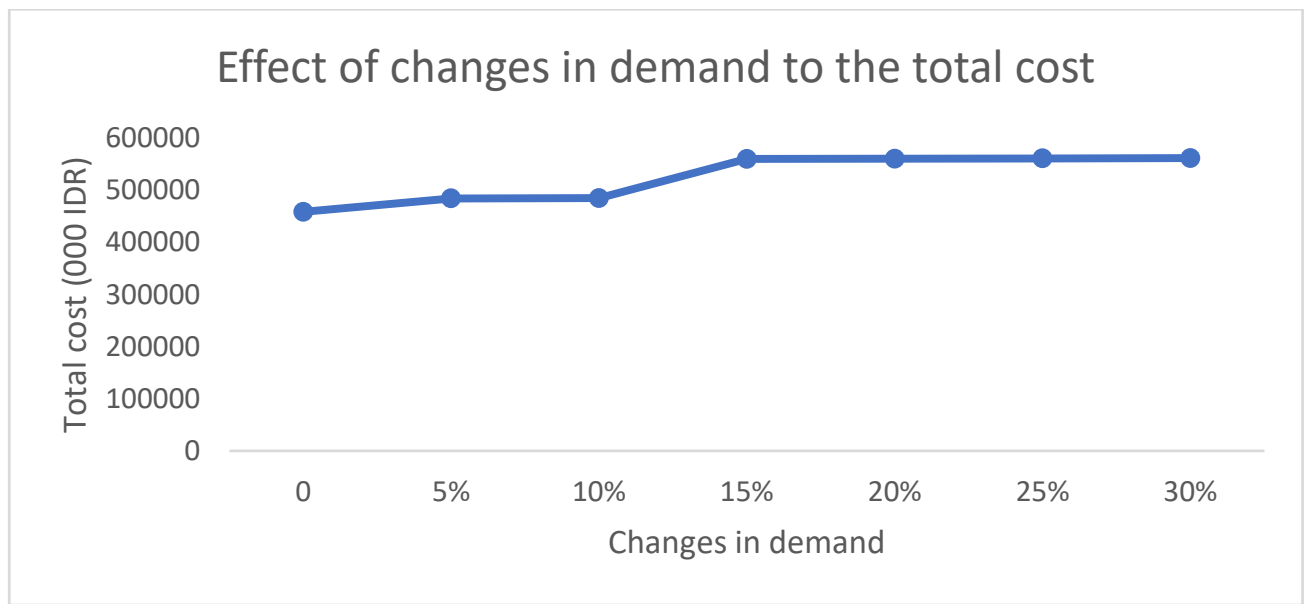

Figure 5. Effect of demand on the total cost

Next, sensitivity analysis is performed to investigate the effect of changes in investment cost to open a distribution center and customer demand on the total cost. The investment costs used in the sensitivity analysis range from -25\%$25 \%$. Meanwhile, the demand used as a sensitivity analysis ranges from $0-30 \%$. For example, the investment cost at location $\mathrm{P} 1$ is IDR $200,000,000$. So, if the investment cost is reduced by $25 \%$, the investment cost becomes
IDR $150,000,000.00$. This value is then used as a parameter. CPLEX is used to find solutions using the new investment value. The same way is conducted for demand. The results of the sensitivity analysis are then presented in Figure 4 and Figure 5.

Figure 4 shows the effect of changes in investment costs on total costs. From Figure 4, the result obtained is a tendency that the graph obtained is a straight line. We can see in the 
second term of equation (18) that transportation costs are a linear function. Therefore, an increase or decrease in investment costs will result in a linear change in total costs.

On the other hand, it is different from the effect of demand on total costs, as shown in Figure 5. An increase in demand up to $10 \%$ graph tends to rise flat. At a $15 \%$ increase in demand, total costs increase substantially. It can be explained as follows. The customer demand is 483 units (data in Table 2). When the demand increase is less than $15 \%$, the total demand is 483 * $1.15<555$ units. The demand for less than 555 units can still be fulfilled by three distribution centers (P1, P2, and P4). However, the demand after passing the threshold of 555 units can only be fulfilled if all suppliers are open. Therefore, the increase in total costs of the $15 \%$ increase in demand was mainly due to increased investment costs. After a $15 \%$ increase, total cost increases occurred, but the increases tended to be flat.

\section{CONCLUSION}

This study developed a supply chain network model to determine the location of distribution centers from an SME in Cilegon. The model is different from other studies due to the non-linear transportation costs. Therefore, the model is transformed into a linear model with the convexcombination method. The model was solved using the CPLEX software.

From the results obtained, the optimal result is to open three suppliers from four possible locations. In addition, the results provide optimal product flow from the production facility to the distribution center and product flow from distribution to customers. According to sensitivity analysis, changes in investment costs against total costs tend to be linear. Meanwhile, changes in demand for total costs are not linear, depending on the supplier's capacity. This research can be developed by considering vehicle emissions to investigate the trade-offs between economic and environmental benefits.

\section{AUTHOR INFORMATION}

\section{Corresponding Authors}

Email: b.kurniawan@untirta.ac.id. Phone: +62 87864902357

\section{Author Contributions}

All authors have contributed equally to this work.

\section{ACKNOWLEDGMENTS}

The authors wish to thank all anonymous referees for constructive feedback.

\section{REFERENCES}

[1] M. Albareda-Sambola, E. Fernández and F. Saldanha-da-Gama, "The facility location problem with Bernoulli demands," Omega, vol. 39, p. 335-345, 2011.

[2] A. Diabat, "A capacitated facility location and inventory management problem with single sourcing," Optimization Letters, vol. 10, p. 1577-1592, 2016.

[3] X. Qi, "Order splitting with multiple capacitated suppliers," European Journal of Operational Research, vol. 178, p. 421432, 2007.

[4] S. Kameshwaran and Y. Narahari, "Nonconvex piecewise linear knapsack problems," European Journal of Operational Research, vol. 192, p. 56-68, 2009.

[5] S. Septiandre and N. Siswanto, "Penentuan Lokasi Gudang Penyangga Regional PT. "X" Wilayah Jawa Timur," Jurnal Studi Manajemen dan Bisnis, vol. 3, p. 184-194, 2016.

[6] A. Montoya, M. C. Vélez-Gallego and J. G. Villegas, "Multi-product capacitated facility location problem with general production and building costs," NETNOMICS: Economic Research and Electronic Networking, vol. 17, p. 47-70, 2016.

[7] G. R. Mauri, F. L. Biajoli, R. L. Rabello, A. A. Chaves, G. M. Ribeiro and L. A. N. Lorena, "Hybrid metaheuristics to solve a multi-product two-stage capacitated facility location problem," International Transactions in Operational Research, 2020.

[8] X. Tang and J. Zhang, "The multi-objective capacitated facility location problem for green logistics," in 2015 4th International Conference on Advanced Logistics and Transport (ICALT), 2015.

[9] A. Estrada-Moreno, A. Ferrer, A. A. Juan, A. Bagirov and J. Panadero, "A biasedrandomised algorithm for the capacitated facility location problem with soft constraints," Journal of the Operational 
Research Society, vol. 71, p. 1799-1815, 2020.

[10] H. Golpîra, "Optimal integration of the facility location problem into the multiproject multi-supplier multi-resource Construction Supply Chain network design under the vendor managed inventory strategy," Expert Systems with Applications, vol. 139, p. 112841, 2020.

[11] M. Salahi and A. Jamalian, "Multi-Source Capacitated Plant Location Problem with Customer and Supplier Matching," Computational Mathematics and Modeling, vol. 26, p. 273-283, 2015.

[12] L.-Y. Wu, X.-S. Zhang and J.-L. Zhang, "Capacitated facility location problem with general setup cost," Computers \& Operations Research, vol. 33, p. 12261241, 2006.

[13] Á. Corberán, M. Landete, J. Peiró and F. Saldanha-da-Gama, "The facility location problem with capacity transfers," Transportation Research Part E: Logistics and Transportation Review, vol. 138, p. 101943, 2020.

[14] A. Diabat and E. Theodorou, "A locationinventory supply chain problem: Reformulation and piecewise linearization," Computers \& Industrial Engineering, vol. 90, p. 381-389, 2015.

[15] P. P. Repoussis and E. J. Alenezy, "Solving Capacitated Facility Location Problem Using Lagrangian Decomposition and Volume Algorithm," Advances in Operations Research, vol. 2020, p. 5239176, 2020.
[16] F. J. F. Silva and D. S. d. I. Figuera, "A capacitated facility location problem with constrained backlogging probabilities," International Journal of Production Research, vol. 45, p. 5117-5134, 2007.

[17] K. Holmberg, "Solving the staircase cost facility location problem with decomposition and piecewise linearization," European Journal of Operational Research, vol. 75, p. 41-61, 1994.

[18] T. R. L. Christensen, K. A. Andersen and A. Klose, "Solving the Single-Sink, FixedCharge, Multiple-Choice Transportation Problem by Dynamic Programming," Transportation Science, vol. 47, p. 428438, 2013.

[19] C. Xin, X. Liu, Y. Deng and Q. Lang, "An optimization algorithm based on text clustering for warehouse storage location allocation," in 2019 1st International Conference on Industrial Artificial Intelligence (IAI), 2019.

[20] A. B. Keha, I. R. d. Farias and G. L. Nemhauser, "A Branch-and-Cut Algorithm without Binary Variables for Nonconvex Piecewise Linear Optimization," Operations Research, vol. 54, p. 847-858, 2006.

[21] M. T. Ramos and J. Sáez, "Solving capacitated facility location problems by Fenchel cutting planes," Journal of the Operational Research Society, vol. 56, p. 297-306, 2005.

[22] J. P. Vielma, S. Ahmed and G. Nemhauser, "Mixed-Integer Models for Nonseparable Piecewise-Linear Optimization: Unifying Framework and Extensions," Operations Research, vol. 58, p. 303-315, 2009. 